\title{
Development of Multi-modal Interfaces in Multi-device Environments
}

\author{
Silvia Berti and Fabio Paternò \\ ISTI-CNR, \\ Via G.Moruzzi 1, 56124 Pisa, Italy \\ \{silvia.berti, fabio.paterno\}@isti.cnr.it
}

\begin{abstract}
Recent technological evolution has enabled environments accessible through a wide variety of interactive devices. Such devices can differ also in terms of interaction modality. In this paper we show how it is possible to generate multi-modal interfaces for different platforms starting with logical user interface descriptions. This approach simplifies the development of applications that can be accessed through a variety of interactive devices and modalities.
\end{abstract}

\section{Introduction}

In recent years, there has been an increasing availability of interactive devices, including mobile phones, personal digital assistants (PDAs), pagers, and so on. This poses a number of challenges for designers and developers of multi-device interfaces. To further complicate this issue, such devices can use different modalities (graphical, vocal, gestural, and so on). Indeed, although several real multimodal systems have been built, their development still remains a difficult task [1].

To address such complexity, a promising solution is to use logical deviceindependent, XML-based [2][4], languages able to represent relevant concepts, such as user tasks and communication goals, along with intelligent transformers able to generate user interfaces in different implementation languages for different platforms depending on their interaction resources, including modalities. Such intelligent rendering can be incorporated in authoring environments to decrease the cost of development of multiple interface versions for the various target platforms, allowing designers to concentrate on the logical decisions without learning a variety of lowlevel detailed implementation languages. However, the design of such transformations has to take into account the features of the target platforms and identify how to exploit the available modalities. Some authors have proposed a component-based approach to ease development of user interfaces [1], exploiting the CARE logical properties for multimodal interfaces. We use logical descriptions that can generate implementations in component-based environments or using other approaches.

In the paper, we discuss how multimodal interfaces (using graphical and vocal modalities) for desktop and PDA can be designed following this approach and show an example application. Lastly, we draw some conclusions and indications for future work. 


\section{The Method}

Our approach exploits a number of transformations that allow designers to move through various views of interactive systems. It allows designers to focus on the logical tasks to accomplish and then transform their descriptions into a user interface description, which incorporates the design decisions but is still in a modality format independent. This is then used to derive a modality-dependent interface description that is used to generate the final code of the user interface. For each logical level considered a XML-based language has been defined. The advantage of this approach is that designers can focus on logical aspects and make design decisions without having to deal with many low-level implementation details. The environment provides support to make concrete design decisions that take into account the target platforms and automatically generate the code where all the low-level details are specified (currently it is able to generate implementations in XHTML, XHTML Mobile Profile, VoiceXML, SVG, $\mathrm{X}+\mathrm{V})$. This type of support is particularly useful when a variety of devices should support access to the interactive application. On the other hand, it is worth noting that in order to make effective decisions designers should be aware of the target platforms and modalities even since the early stages of the design process. They do not need to know the implementation details of all the targeted devices but they still should know their main features (through the platform concept).

Indeed, there are tasks that may be meaningful only when using some specific platform or modality. For example, watching a long movie makes sense only in a multimedia desktop system whereas accessing information from a car in order to know directions to avoid a traffic jam can be done only through a mobile device and if this task should be performed while driving it can be supported only through a vocal interface. The available modality may also have an impact on how to accomplish a task, for example a vocal interface or a graphical mobile phone interface can require to perform sequentially tasks that can be performed concurrently in a desktop graphical interface.

In our approach a user interface is structured into a number of presentations. The presentation is structured into interactors (logical descriptions of interaction techniques) and composition operators that indicate how to put together such interactors. While at the abstract level such interactors and their compositions are identified in terms of their semantics in a modality independent manner, at the concrete level their description and the values of the attributes depend on the available modality. When both vocal and graphical support are available, the multimodality can be exploited in different manners. The modalities can be used alternatively to perform the same interaction. They can be used synergistically within one basic interaction (for example, providing input vocally and showing the result of the interaction graphically) or within a complex interaction (for example, filling in a form partially vocally and partially graphically). Some level of redundancy can be supported as well, for example when feedback of a vocal interaction is provided both graphically and vocally.

The composition operators are associated with a communication goal. A communication goal is a type of effect that designers aim to achieve when they want to structure presentations. Grouping is an example of composition operator that aims to highlight the logical relation amongst a group of interface elements. It can be 
implemented in the graphical channel through one or multiple attributes (fieldset, colour, position...), whereas in the vocal channel it is possible to group elements by inserting a sound or a pause at the beginning and the end of the grouped elements. In case of multimodal interfaces we have to consider the actual resources available for the modalities. Designing a multimodal interface for a desktop is different from designing one for a PDA because the graphical resources available are different. The composition operators such as grouping are implemented graphically in desktop interfaces because the graphical modality is dominant, whereas in mobile devices they are supported both graphically and vocally.

If we consider the interactors the reasoning is similar. In the case of multimodal interfaces for a desktop platform we have decided to employ the graphical representation for only-output interactors, because the interface has a lot of space available, while for PDAs we have decided to use either modality or both in a complementary manner depending on the specific case (for example, the vocal modality is used for long texts). In interactive elements, in the case of desktop the prompt, input, and feedback are graphical (and the input can be also vocal), whereas in the case of PDA in addition the prompt can be vocal and the feedback is also always vocal unless the designer specifies otherwise.

\section{A Sample Application and Concluding Remarks}

In this section we show an example multimodal application for a multi-hall cinema designed with our environment. This example aims to highlight different and similar aspects between a PDA and a desktop multimodal interface. In both versions users can check the movies currently being shown or coming soon and book a ticket.

The main difference is that in the PDA version the vocal modality is more used than in the desktop version because handheld devices have limited graphics capabilities, and a small screen. As you can see in Figure 1, the movie description page of the desktop version provides more information, for example "user rating" or the film trailer. In addition, all tasks that do not require user interaction (only output interactors) are implemented using graphical modality. Differently, in the PDA version some tasks are not supported because the PDA screen is small and it is very tedious to scroll down so much, and some more important tasks are supported in the vocal modality, for example the plot summary. When users access the film description page with the PDA, they start to listen a little summary while some general information is shown. It is also interesting to highlight that users can provide input through either graphical or vocal modalities. In both versions it is possible to access the booking page using vocal commands or graphical selection. In case there are two or more input interactors in the same presentation, users can provide all information with only one vocal command or can select graphically each object. For example, in the booking page the user can say the time and the hall number together ("I would like to book a ticket for a screening in the Hall 3 at 9 p.m.") or can select graphically the two options. This features is very useful when users interact with PDAs, where there is no keyboard, because the interaction becomes much faster. In this example you can notice the different techniques adopted for grouping elements: in the multimodal desktop interface it is obtained through a graphical field set, 
whereas in the PDA version is obtained using both a sound (for the vocal comment) and a background colour at the same time to delimit the grouped elements.

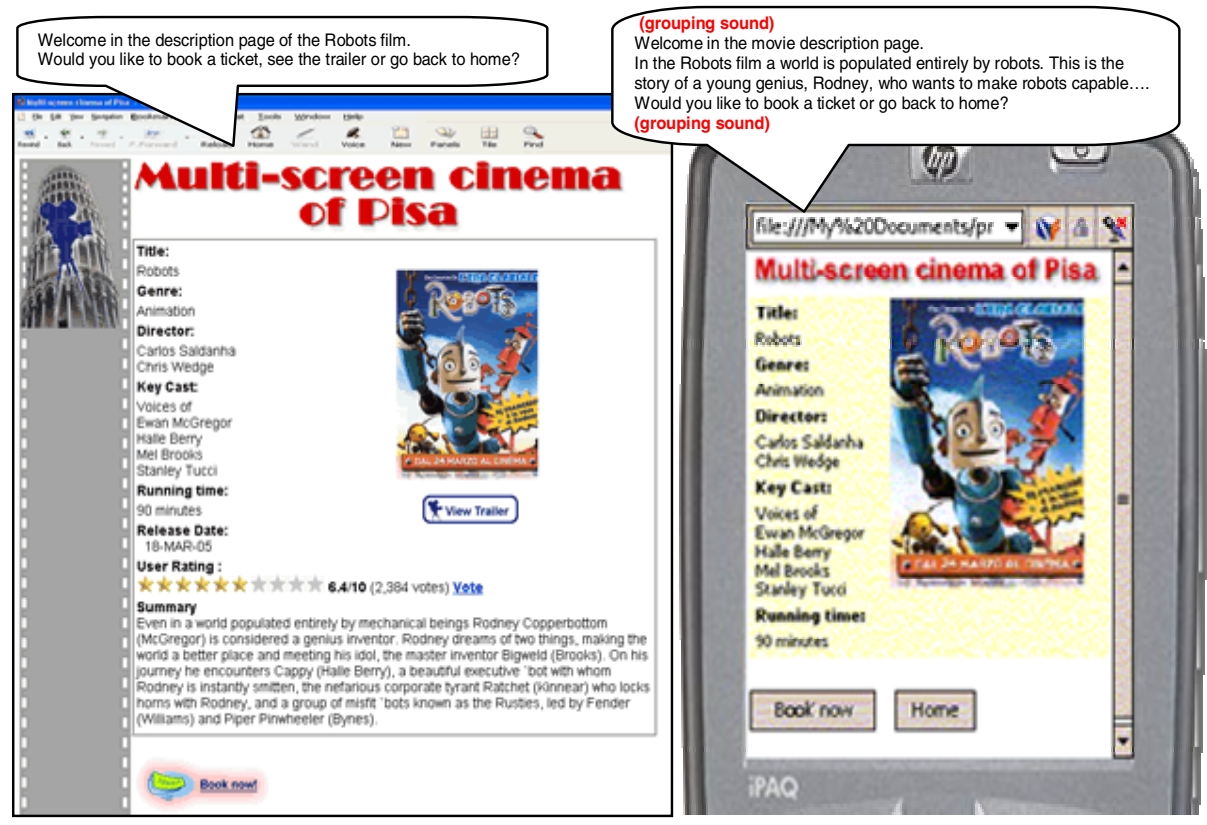

Fig. 1. Two multi-modal versions (desktop and PDA) for the cinema application

We have presented an environment supporting design of multimodal interfaces in multi-device environments. In particular, we have considered development of graphical and vocal interfaces in desktop and PDA devices. The approach is based on the use of logical, device-independent descriptions and transformations that incorporate multi-modal design criteria. An example application has been shown. Future work will be dedicated to extending the approach to consider other modalities, such as gestural interaction.

\section{References}

1. Bouchet J., Nigay L., ICARE: A Component-Based Approach for the Design and Development of Multimodal Interfaces, Adjunct Proceedings CHI 2004, pp.1325-1328.

2. Mori, G., Paternò, F., Santoro, C., Design and Development of Multi-Device User Interfaces through Multiple Logical Descriptions, IEEE Transactions on Software Engineering, August 2004, Vol.30, N.8, pp.507-520, IEEE Press.

3. Coutaz, J. Nigay, L. Salber, D. Blandford, A. May, J. Young, R.,. Four Easy Pieces for Assessing the Usability of Multmodal Interaction: the CARE properties. Proceedings INTERACT 1995, pp.115-120

4. Puerta, A., Eisenstein, XIML: A Common Representation for Interaction Data, Proceedings ACM IUI'01, pp.214-215. 